\title{
Construction of the Real Number System
}

\author{
Reema Agarwal $^{1}$, Mahesh Kumar ${ }^{2}$ \\ 1(Department of Mathematics, Lady Shri Ram College For Women, University of Delhi, India) \\ 2(Department of Mathematics, Lady Shri Ram College For Women, University of Delhi, India)
}

\begin{abstract}
In this paper it will be shown that $\mathbb{R}$ is the only complete Archimedean ordered field. The two approaches to completeness for Archimedean ordered field are given and then it is concluded that they are equivalent.
\end{abstract}

Keywords: Complete Archimedeanordered field, right end point, open lower segment.

\section{Defect In Rationals}

Althoughit has beennoticedthatthesetof rationals $\mathbb{Q}$ formsa richalgebraic systemhavingorderproperties, itisinadequateforthepurposeofanalysis. It hasalready beennoticed that not every positive rational number has a rational square root. For example,there is norational number whose square is 2 . The defect in the rationalsthat has been described here may be described in a variety of ways. One form of the defect that hasalready beennoticed is that a nonempty subset of rational numbers that is bounded above need not have a least upper bound (in the set of rationals). A slightyless standard approach which is more picturesque is that there are open lower segments in $\mathbb{Q}$ without havingrightendpointsin $\mathbb{Q}$,andthis isconsideredtobea defect.Forthis purpose,thenotionofanopenlowersegmentinthesetofrationals is defined.

Definition 1:Aset $J \subset \mathbb{Q}$ iscalledanopenlowersegmentif

1) $J \neq \Phi$

2) $J \neq \mathbb{Q}$

3) Forevery $x \in J$,thereisay $\in J$ suchthat $y>x$,

4) If $x \in J$ and $y<x$ then $\in J$.

Apoint $x$ willbecalledtherightendpointofanopenlowersegmentJif

1) forevery $y \in J, x>y$,

2) ifzissuchthatforevery $y \in J, z>y$, then $z \geq x$.

For example, consider $J \subset \mathbb{Q}$ to be the set of all non-positive rational numbers, together withthepositive rational numbers whosesquareisless than2.ThenJisanopenlowersegmentof $\mathbb{Q}$ havingnorightendpointin $\mathbb{Q}$.

Note:IfarightendpointofJexists, itisunique, andisthesmallestrational greaterthaneveryelementofJ.

\section{The Real Numbers}

The set of real numbers is an extension of the set of rational numbers, whichremovesthedefectdescribedabove.It will beseenthattherealnumbers are an Archimedean ordered field in which every open lower segment has a right end point. The real numbers are obtained from the rationalsby what may be described as filling the gaps. In otherwords, the rightendpoints are addedasidealelements, ornewnumbers, tocorrespondtothose openlowersegmentswhichdonothaverightendpointsamongtherationals.

$$
\text { Onewayof doingthisistoconsidertheopenlowersegmentitselftobea }
$$

substitute foritsownrightendpoint.Onecanseethatthisisanentirely natural approach when one agrees that open lower segments aretobein one-to-onecorrespondencewiththeirrightendpoints.

Inaccordancewiththeabove, theset $\mathbb{R}$ ofrealnumbersisdefinedtobethe setofopenlowersegmentsofrationalnumbers.

Firstaddition is defined in $\mathbb{R}$.

Let $I$ and $J$ berealnumbersi.e.,openlowersegmentsofrationalnumbers. Define $I+J$ as

$$
I+J=\{x+y: x \in I, y \in J\}
$$

Then $I+J$ isanopenlowersegment.Let $x \in I$ and $y \in J$, so that $x+y \in I+J$, and let $u<x+y$. Then we can write $u=x+z$, where $x \in I$ and $z(=u-x)<y$, so that $z \in J$. So,$x+z>x+y$ and $x+z \in I+J$. Now, it follows that $I+J$ is an open lower segment. Also 
andthat

$$
I+J=J+I
$$

$$
(I+J)+K=I+(J+K)
$$

follows directly from the definition of ' + 'in $\mathbb{R}$ and commutative and associativepropertiesof $\mathbb{Q}$.

Let $\mathcal{O}$ betheopen lowersegment ofnegative rational numbers. One canverifythat

$$
I+\mathcal{O}=\text { Iforall } I \in \mathbb{R}
$$

Finally,theequation

$$
I+X=J
$$

hasasolutionforevery $I, J \in \mathbb{R}$.Let $X$ consistofall $x$ such

that $y+x \in J$ foreveryy $\in$

$I$, exceptforthelargestsuch $x$ ifthereisone.

Thesolutionof $I+X=\mathcal{O}$ isdesignated $-I$.

AnorderrelationisintroducedinRbyletting

$$
I>\text { Jif } I \supset J
$$

Thus Rbecomesanorderedset.ClearlyI > OifandonlyifI containsa positiverational.

Nowmultiplication is definedin $\mathbb{R}$.If $I>\mathcal{O}, J>\mathcal{O}$ define $I J$ asthe setofallnonpostiverationalstogetherwithall $x y$ where $x \in I, x>0$, and $y \in J, \mathrm{y}>0$

If $I=\mathcal{O} \quad$ or $\quad J=\mathcal{O}$ define $I J=\mathcal{O}$.Ifboth $I<O$ and $J<O$ define

IfexactlyoneofI, Jislessthan $\mathcal{O}$, say $I<\mathcal{O}$,define

$$
I J=(-I)(-J)
$$$$
I J=-(-I)(J)
$$

Nowonecanshowthat $\mathbb{R}$ isanorderedfield.Thecompletedetailscanbe foundin[2].

Moreover, RisArchimedean.Let $I>O, J>\mathcal{O}$.Thenthereexistsapositive $\quad$ rational $x \in I$ andan $n \in \mathbb{N}$ suchthat $x \notin$ $J$,since $\mathbb{Q}$ is Archimedeanand $I, J$ areopenlowersegments.Thus, itfollowsfromthetrichotomyproperty of $\mathbb{R}$ that $n I>$ $J$.

Then $J \in \mathbb{R}$ forwhich/hasarationalrightendpointareinone-to-one correspondence withtherationalnumbers, theassociatedmappingisorder preservingandadditionandmultiplicationpreserving.

Thus, $\mathbb{Q}$ isimbeddedin $\mathbb{R}$,orthat $\mathbb{Q}$ isisomorphictoanorderedsubfieldof $\quad \mathbb{R}$.Thissubfieldis $\quad$ called therationalnumbersandhencesmallletters will be used forelementsofRinTheorem2.

Nowopenlowersegmentsofrealsandtheirrightendpoints can be defined inthesamewayasforrationals.

Theorem1:Everyopenlowersegmentofrealshasarightendpoint.

Proof: Let $\mathcal{J}$ beanopenlowersegmentofreals.Let

$$
U=\bigcup\{J: J \in \mathcal{J}\}
$$

Weshowthat $U$ isanopen lower segment ofrationalsi.e., $U \in \mathbb{R}$.

Let $x \in U$.Thenthereisa $J \in \mathcal{J}_{\text {suchthat }} x \in J$. For every $y<x, y \in J \subseteq U$. Alsothereisay $\in J \subseteq U$ suchthat $y>x$. Moreover, thereisan $I \notin \mathcal{J}$ since $\mathcal{J}$ isan open lower segment ofreals. Then $x \notin$ Iimplies $x \notin U$ because if $x \in U$ then $x \in J$ for some $J \in \mathcal{J}$ which implies $I \subset J$ and hence $I \in \mathcal{J}$, a contradiction. Thus $U$ is an open lower segment of rationals. Next it is shown that $U$ is the right end point of $\mathcal{J}$.By definition of $U, U>J$ for every $J \in \mathcal{J}$. Suppose $V>J$ for every $J \in \mathcal{J}$.Then $V \geq U$. Hence, $U$ is the right end point of $\mathcal{J}$.

NowanotherformofTheorem1 is given whichisreferredtoastheleastupper boundproperty(orcompleteness propertyinthesenseofDedekind) ofthe setofrealnumbers $\mathbb{R}$.

Theorem2:IfS $\subset \mathbb{R}$ isnonemptyandhasanupperbound,ithasaleast upperbound.

Proof: Ifthegivenupperboundbelongsto $S$ thenthatwillonlybetheleast oneandwearedone. Soweassume thatnoupper boundfor $\operatorname{Sisin} S$. Now we define a set $U$ by letting $x \in U$ if and only if there is ay $\in S$ such that $y>x$. We show that $U$ is an open lower segment of reals. If $x \in U$ and $z<x$ then $z \in U$ by definition of $U$. Also, if $x \in U$ then there is a $z \in U$ such that $z>x$ since no upper bound for $S$ is in $S$. Moreover, every upper bound of $S$ is not in $U$. Thus, Uisanopenlowersegmentofreals, andsoithasarightendpoint, sayubyTheorem1. Next it is shownthat $u=\sup S$.Let $x \in S$. Then $y<x$ implies $y \in U$ sothat $y<u$.Hence $x \leq u$ (since 
$\mathrm{u}<\mathrm{x} \Rightarrow \mathrm{u} \in$ Uwhichisacontradiction).Thus, uisanupperboundofS .

Lety $<u$.Theny $\in$ UsinceUisanopenlowersegmentanduisitsright

endpoint.SothereisanxinSwithy $<x$.Thus, yisnotanupperbound ofS.Henceu $=$ supS .

Thus, itisactuallythepropertyofTheorem1 thatisbeingusedtoobtain allfurtherpropertiesof therealnumbers. Thus, thefactthatRisa complete Archimedeanorderedfieldisbasictoallfurtherdevelopments.

The Theorem 3 below shows that $\mathbb{R}$ is the only complete Archimedean orderedfield.

Theorem3:AnytwocompleteArchimedeanorderedfields $F_{1}$ and $F_{2}$, with setsofpositiveelements $P_{1}$ and $P_{2}$, respectively,arealgebraicallyandorder isomorphic, i.e.,thereexists aone-to-one mapping $\tau$ of $F_{1}$ onto $F_{2}$ such that

$\tau(x+y)=\tau(x)+\tau(y), \tau(x y)=\tau(x) \tau(y), \tau(x) \in P_{2}$ iff $x \in P_{1}$.

Proof:

Let $1_{1}$ and $1_{2}$ betheunitsof $F_{1}$ and $F_{2}$ and $0_{1}$ and $0_{2}$ thezeros.

Notethateveryorderedfieldcontainsanisomorphof $\mathbb{Q}$.Sowedefine

the

mapping $\tau$ firstontherationalelementsof $F_{1}$ asfollows.

wheremisaninteger, $n$ isanonzerointeger

$$
\tau\left(\frac{m}{n} 1_{1}\right)=\frac{m}{n} 1_{2}
$$

If $x \in F_{1}$ and $x$ isnotoftheform,$\frac{m}{n} 1_{1}$ then define

Onecanprovethat $\tau$ hasthedesiredproperties.

$$
\tau(x)=\sup \left\{\frac{m}{n} 1_{2}: \frac{m}{n} 1_{1}<x\right\}
$$

Nowanotherconstructionof therealnumbersystem is given inwhicha real numberisdefined asanequivalenceclassofCauchysequencesofrational numbers.

Definition $2:$ Let $F$ beanorderedfield. A sequence $\left(a_{n}\right)$ of elements of $F$ is called bounded if there is an element $b \in F$ such that $\left|a_{n}\right| \leq b$ for each positive integer $n$.

Definition 3: Asequence $\left(a_{n}\right)$ ofelements of $F$ iscalled Cauchy iffor every $e \in F$ such that $e>0$, there is a positive integer $N$ such that $\left|a_{p}-a_{q}\right|<e$ for all $p, q \geq N$.

Definition $\quad 4$ :Asequence $\left(a_{n}\right)$ ofelementsof $F$ iscallednullifforevery

0 , thereisapositiveinteger $N$ suchthat, $\left|a_{p}\right|<e$ for all $p \geq N$.

$e \in F$ suchthate $>$

Thefamiliesofsequencessatisfyingtheseconditionswillbedenoted by $\mathcal{B}, \mathcal{C}$ and $\mathcal{N}$ respectively.

Now fewtheorems and lemmas (without proofs) are statedbefore stating thetwoimportantresultswhichconcernthemainthemeof thispaper.The detailsoftheproofscanbefoundin[3].

Theorem4:Theinclusions $\mathcal{N} \subset \mathcal{C} \subset \mathcal{B}$ isobtained.

Theorem 5: For $\left(a_{n}\right),\left(b_{n}\right) \in \mathcal{C}$,let $\left(a_{n}\right)+\left(b_{n}\right)=\left(a_{n}+b_{n}\right)$ and $\left(a_{n}\right)\left(b_{n}\right)=\left(a_{n} b_{n}\right)$. With these definitions of sum and product, $\mathcal{C}$ is a commutative ring with unity, and $\mathcal{N}$ is an ideal in $\mathcal{C}$ such that $\mathcal{N} \subsetneq \mathcal{C}$.

Theorem6:Let $\mathcal{C} / \mathcal{N}$ denotethesetwhoseelementsaretheset $\left(a_{n}\right)+\mathcal{N}$ (called cosets of $\mathcal{N}$ ), where $\left(a_{n}\right) \in \mathcal{C}$. Addition and multiplication in $\mathcal{C} / \mathcal{N}$ are defined by

$\left(\left(a_{n}\right)+\mathcal{N}\right)+\left(\left(b_{n}\right)+\mathcal{N}\right)=\left(a_{n}\right)+\left(b_{n}\right)+\mathcal{N}=\left(a_{n}+b_{n}\right)+\mathcal{N}$ and

$\left(\left(a_{n}\right)+\mathcal{N}\right)\left(\left(b_{n}\right)+\mathcal{N}\right)=\left(a_{n}\right)\left(b_{n}\right)+\mathcal{N}=\left(a_{n} b_{n}\right)+\mathcal{N}$

Thesedefinitionsareunambiguous, andwithadditionandmultiplicationso defined, $\mathcal{C} / \mathcal{N}$ isafield. 
Notation: The field $\mathcal{C} / \mathcal{N}$ will be written as $\bar{F}$. Henceforth elements $\left(a_{n}\right)+\mathcal{N}$ of $\mathcal{C} / \mathcal{N}$ will be denoted by small greek letters: $\alpha, \beta, \ldots \ldots$. If $a \in F$ then the element $\left(a_{n}\right)+\mathcal{N}$ of $\bar{F}$ will be written as $\bar{a}$; it is the coset of $\mathcal{N}$ containing the constant sequence all of whose terms are $a$.

Theorem 7: In $\bar{F}$, let $\bar{P}=\left\{\alpha \in \bar{F}: \alpha \neq \overline{0}\right.$ andthereexists $\left(a_{n}\right) \in \alpha$ such that $a_{n}>0$ for $\left.\mathrm{n}=1,2, \ldots \ldots\right\}$. With this set $\bar{P}, \bar{F}$ is an ordered field. The mapping $\tau: \tau(a)=\bar{a}$ is an order preserving algebraic isomorphism of $F$ into $\bar{F}$.

Definition 5: Given a sequence $a_{n}$ in an ordered field $F$ and $b \in F$, it is said that limit of $a_{n}$ is $\mathrm{b}$ and we write $\lim _{n \rightarrow \infty} a_{n}=b$ or $a_{n} \rightarrow b$ if for every positive $e$ in $F$ there exists a positive integer $L$ such that $\left|a_{n}-b\right|<e$ for all $n \geq L$. An ordered field is said to be complete (in the sense of Cantor) if every Cauchy sequence in $F$ has a limit in $F$.

Lemma 1: A sequence with a limit is a Cauchy sequence. If $\left(a_{n}\right)$ is a Cauchy sequence and $\left(a_{n_{k}}\right)$ is a subsequence with limit $\mathrm{b}$, then $\left(a_{n}\right)$ has limit $\mathrm{b}$.

Lemma 2: For $\alpha>0, \alpha \in \bar{F}$, there exists $e \in F$ such that $\overline{0}<\bar{e}<\alpha$. If $F$ is Archimedean ordered,then $\bar{F}$ is also Archimedean ordered.

Lemma 3: Let $\alpha \in \bar{F}$ and $\left(a_{n}\right) \in \alpha$. Then $\lim _{n \rightarrow \infty} \bar{a}_{n}=\alpha$.

Now the two important results mentioned above are stated.

Theorem 8: The field $\bar{F}$ is complete (in the sense of Cantor).

ThefollowingTheorem 9

belowshowsthatacompleteArchimedeanordered

field

(completeinthesenseofCantor)isalsocompleteArchimedeanordered field(completeinthesenseofDedekind).

Theorem9:Let $F$ beacompleteArchimedean

thesenseofCantor),andlet $A$ beanonemptysubsetof $F$ thatisbounded above.Thensup Aexists.

orderedfield(completein

Note:Theorem3abovealsoholdsforcompleteArchimedeanorderedfields(completeinthesenseofCantor).So,wehavet hefollowingdefinition:

Definition 6: Therealnumber fieldRisanycomplete ordered field.For example, $\overline{\mathbb{Q}}$.

III. Conclusion:

We see that the two approaches to completeness for Archimedean orderedfieldsareequivalent.So,fortherealsitisentirelya matterof choicewhichapproachoneprefers.However,therearesituationsin

whichtheCauchysequenceapproachistheonlyonepossible.Forexample, in the field of complex numbers $\mathbb{C}$ which is not ordered $\left(a s i \neq 0\right.$ buti $\left.^{2}=-1<0\right)$ Theorem 8 which is another versionof the completenessproperty for fields does not require the order relation, $<$. It is a useful axiom toconsiderforotherfieldsotherthanorderedfields. Allthatisrequiredis thedistancefunctiond $(x, y)$ tohavemeaninginthatfield.

\section{Books:}

\section{Reference}

[1] CasperGoffman,IntroductiontoRealAnalysis

[2] W.Rudin,Principle ofMathematical Induction

[3] HewittandStromberg, RealandAbstractAnalysis

[4] N.L.Carothers, RealAnalysis 